\title{
Effects of delayed HIF-1 $\alpha$ expression in astrocytes on myelination following hypoxia-ischaemia white matter injury in immature rats
}

\author{
Min-Jie Wang ${ }^{1,2 \#} \wedge$, Zhi-Hua Li ${ }^{1,2 \#}$, Rui-Wei Gao ${ }^{1,2}$, Qiu-Fan Chen ${ }^{2}$, Jie Lin ${ }^{2}$, Mi-Li Xiao ${ }^{1,2}$, Ke Zhang ${ }^{1,2}$, \\ Chao Chen ${ }^{1,2}$ \\ ${ }^{1}$ Department of Neonatology, Children's Hospital of Fudan University, Shanghai, China; ${ }^{2}$ Key Laboratory of Neonatal Disease, Ministry of Health, \\ Shanghai, China \\ Contributions: (I) Conception and design: C Chen, MJ Wang; (II) Administrative support: C Chen, ZH Li; (III) Provision of study materials or \\ patients: MJ Wang, RW Gao, QF Chen; (IV) Collection and assembly of data: MJ Wang, J Lin; (V) Data analysis and interpretation: ZH Li, ML \\ Xiao, K Zhang; (VI) Manuscript writing: All authors; (VII) Final approval of manuscript: All authors. \\ \#These authors contributed equally to this work. \\ Correspondence to: Chao Chen, PhD. Department of Neonatology, Children's Hospital of Fudan University, No. 399, Wanyuan Road, Minhang \\ District, Shanghai 201102, China. Email: chaochen@fudan.edu.cn.
}

Background: The underlying cause of neurological sequelae after immature cerebral hypoxia-ischaemia (HI) white matter injury is impaired myelination. Previous studies have indicated that astrocyte activation is closely related to impaired myelination. However, the mechanism of reactive gliosis in white matter injury post-HI remains poorly understood.

Methods: Studies using adult ischaemic animal models demonstrated that hypoxia inducible factor$1 \alpha(\mathrm{HIF}-1 \alpha)$ expression was involved in the formation of reactive astrocytes. Here, we investigated the temporal expression of HIF-1 $\alpha$ and its impact on reactive gliosis and further myelination using a perinatal $\mathrm{HI}$ white matter injury model induced in rats at postnatal day 3 . The temporal pattern of HIF-1 $\alpha$ expression post-HI injury was tested by western blotting and immunofluorescence. Rats were treated with a HIF-1 $\alpha$ inhibitor at 72 hours post-HI injury. Reactive gliosis and myelination were assessed with western blotting, immunofluorescence and electron microscopy, and neurological functions were examined by behavioural testing.

Results: Our results showed that the expression of HIF-1 $\alpha$ was upregulated in neurons at 24 hours and in astrocytes at 7 days post-HI. Inhibiting delayed HIF-1 $\alpha$ expression post-HI injury could restrain reactive gliosis, ameliorate hypomyelination, and improve the performance of rats in the Morris water maze test.

Conclusions: Our findings suggest that a delayed increase in HIF-1 $\alpha$ in astrocytes is involved in glial scar formation and leads to arrested oligodendrocyte maturation, impaired myelination, and long-term neurological function after experimental white matter injury in immature rats.

Keywords: White matter injury; myelination; astrogliosis; oligodendrocytes; hypoxia inducible factor-1 $\alpha(\mathrm{HIF}-1 \alpha)$

Submitted Aug 29, 2021. Accepted for publication Dec 15, 2021.

doi: $10.21037 / \mathrm{tp}-21-407$

View this article at: https://dx.doi.org/10.21037/tp-21-407

^ ORCID: 0000-0001-9389-0687. 


\section{Introduction}

Preterm infants are particularly susceptible to cerebral white matter injury, especially those with a gestational age of less than 28 weeks (1), while their mortality has decreased with the development of neonatal care (2). Advances in the care of preterm infants have resulted in a sustained reduction in the severity of injuries, which has resulted in a shift from more severe focal necrotic lesions to milder diffuse and microscopic white matter injury related to disrupted functional connectivity and microstructure $(3,4)$. Previous studies have demonstrated that altered functional network connectivity, especially in the corpus callosum area, lasts until adolescence and correlates with a broad spectrum of cognitive and neurological deficits (5-7).

The key stage of the development of white matter, especially the proliferation and differentiation of oligodendrocyte precursor cells (OPCs) and immature oligodendrocytes, in rodents is mainly during the first 2 postnatal weeks, whereas in humans, it is mostly between 23 and 32 weeks of gestation $(8,9)$. Given the perinatal asphyxia, respiratory and circulatory failure and other complications induced by immature lung and heart development, the brains of preterm infants at the gestational age of 23-32 weeks are more likely to suffer hypoxia and ischaemia. Due to the maturation-dependent vulnerability of the oligodendrocyte lineage to hypoxiaischaemia (HI), OPCs are particularly susceptible to $\mathrm{HI}$ injury and die during the early phase of injury, followed by rapid regeneration $(9,10)$. However, these regenerated OPCs display arrested maturation and fail to differentiate into mature oligodendrocytes, resulting in abnormal myelination $(9,11)$.

Many studies have shown that reactive astrocytes have an impact on oligodendrocyte maturation through a paracrine mechanism involving cytokines $(12,13)$ and macromolecular substances that constitute the extracellular matrix, such as chondroitin sulphate proteoglycans (CSPGs) (14). Our previous work demonstrated that enhanced degradation of CSPGs in the white matter region via chondroitinase $\mathrm{ABC}$ (cABC) could ameliorate hypomyelination after $\mathrm{HI}$ injury in immature rats (15). However, the mechanism of reactive gliosis in white matter injury post-HI remains poorly understood.

Hypoxia inducible factor- $1 \alpha(\mathrm{HIF}-1 \alpha)$ is a master transcription factor controlling oxygen homeostasis and is essential in cellular adaptation to hypoxia (16). Many studies have shown the strong neuroprotective effect of
HIF-1 $\alpha$ against hypoxia and ischaemia, but most of them have focused on neurons. Given the pathology of white matter injury in the immature brain, the role of HIF-1 $\alpha$ in neuroglia should be different. A neonatal mouse model of chronic hypoxia found that constitutive HIF-1 $\alpha$ stabilization resulted in OPC maturation arrest (17). Some studies examining astrocytes using adult ischaemic animal models demonstrated that HIF-1 $\alpha$ expression was involved in the formation of reactive astrocytes $(18,19)$.

In this study, we hypothesized that HIF-1 $\alpha$ was also involved in reactive gliosis in our $\mathrm{HI}$ white matter injury model of immature rats, contributing to arrested OPC maturation and impaired myelination. However, HIF-1 $\alpha$ still plays an important role in the adaptation of cells to hypoxia, indicating that it may play different roles in a timedependent manner in white matter injury post-HI.

We present the following article in accordance with the ARRIVE reporting checklist (available at https:// tp.amegroups.com/article/view/10.21037/tp-21-407/rc).

\section{Methods}

\section{Animals}

Experiments were approved by the Research Ethics Board of the Children's Hospital of Fudan University (No. 2016-06), and rats were treated in accordance with the National Institutes of Health (NIH) Guide for the Care and Use of Laboratory Animals. Pregnant female Sprague-Dawley rats at gestational day 16 were purchased from Sippr-BK Laboratory Animal Limited Company (Shanghai, China) and maintained in temperature-, humidity-, and lightcontrolled conditions with free access to food and water. Following delivery, litter sizes were adjusted to 10-12 pups per litter to normalize pup weights. Pups were raised by the dam before and after modelling.

\section{White matter injury of immature rats}

White matter injury of immature rats was induced in 3 -day-old SD rats $(\mathrm{P} 3$, day of birth $=\mathrm{P} 0)$ as previously described (20). Briefly, the littermate pups were assigned to injury group and sham group with balanced sex-ratio and weights. The pups assigned to injury group were underwent right common carotid artery ligation after anaesthetization with isoflurane. After a recovery period of $90 \mathrm{~min}$, the pups were placed in a closed containers that were submerged in a water bath to maintain the temperature at $37^{\circ} \mathrm{C}$. To induce 
moderate injury, we perfused the container with a mixture of nitrogen and oxygen with a concentration of $6 \%$ oxygen for $150 \mathrm{~min}$. The littermate sham pups underwent isolation of the right common carotid artery without ligation or exposure to hypoxia. The pups were randomly assigned to experimental groups after injury.

\section{Drug administration}

We used 2-methoxyestradiol (2-ME) (Selleck, USA) to inhibit HIF-1 $\alpha$. The timing of administration was selected based on the expression pattern of HIF-1 $\alpha$ post-HI injury in the corpus callosum, and the dose and route were tested and determined as described elsewhere (21) and according to the survival rate of the pups. The protocol for drug administration was as follows. First, 2-ME was dissolved in a mixture solution of $70 \%$ saline and $30 \%$ dimethylsulfoxide (DMSO) and applied at a dose of $5 \mathrm{mg} / \mathrm{kg}$ by intraperitoneal injection at $72 \mathrm{~h}$ after $\mathrm{HI}$ injury according to the grouping $(\mathrm{C}+\mathrm{M} 3$ and $\mathrm{H}+\mathrm{M} 3)$. The blank groups received intraperitoneal injections of a mixture of $70 \%$ saline and $30 \%$ DMSO at the same volume and time $(\mathrm{C}+\mathrm{B} 3$ and $\mathrm{H}+\mathrm{B} 3)$.

\section{Western blotting}

For western blotting, rats at different time points postHI injury were sacrificed after deep anaesthetization, and the right corpus callosum was dissected carefully. Tissue was lysed by a tissue lyser (JingXin, China) in T-PER tissue protein extraction reagent (78510) containing Halt protease inhibitor cocktail (87785) according to the manufacturer's instructions (Thermo, USA). Protein concentrations were determined using a BCA Protein Assay kit (Beyotime, China). Equal amounts of protein extract were resuspended in SDS-PAGE sample loading buffer (Beyotime), separated by SDS-PAGE and transferred to nitrocellulose membranes. Nonspecific protein binding was blocked by incubation of nitrocellulose sheets in Trisbuffered saline containing $0.1 \%$ Tween-20 (TBST) and 7\% nonfat milk for $2 \mathrm{~h}$ at room temperature. The membranes were then incubated with the following primary antibodies: HIF-1 $\alpha$ (1:500; Novus Biologicals, USA), MBP (1:1,000; BioLegend, USA) and $\beta$-actin (1:5,000; OriGene, USA) overnight at $4{ }^{\circ} \mathrm{C}$. The following day, the membranes were incubated with HRP-conjugated goat anti-mouse antibody (Absin, China) at room temperature for $1 \mathrm{~h}$ after three 15 min washes with TBST and then rinsed three times with TBST for $15 \mathrm{~min}$. Immunoreactivity was detected by enhanced chemiluminescence (ECL) detection using the SuperSignal kit (Thermo, USA) according to the manufacturer's instructions.

\section{Histology and immunofluorescence}

Rats were deeply anaesthetized and perfused transcardially with saline followed by $4 \%$ paraformaldehyde (PFA). Whole brains were rapidly dissected and postfixed at $4{ }^{\circ} \mathrm{C}$ in $4 \%$ PFA and in a solution containing 4\% PFA and 20\% sucrose for $24 \mathrm{~h}$ each and then immersed in a solution containing $30 \%$ sucrose in PB. For frozen sections, the brains were frozen in an embedding Tissue-Tek OCT matrix (Sakura Finetek, USA) at $-80^{\circ} \mathrm{C}$, and coronal sections $(30 \mu \mathrm{m})$ were prepared using a cryostat (CM1950, Leica, Germany) and cryoprotected in solutions containing $30 \%$ sucrose and $30 \%$ ethanediol in PB. For histological examination, the sections were stained with haematoxylin and eosin and imaged using a stereomicroscope (SZX16, Olympus, USA). For immunofluorescence, the sections were fixed with $4 \%$ PFA for 10 min, permeabilized with $0.2 \%$ Triton X-10 and treated with $10 \%$ donkey serum in PBS for $1 \mathrm{~h}$ to block nonspecific binding. The sections were incubated overnight at $4{ }^{\circ} \mathrm{C}$ with the following primary antibodies developed in different species and diluted in 5\% donkey serum: mouse anti-MBP (1:200; BioLegend), rabbit anti-NFH (1:5,000; Abcam, UK), mouse anti-HIF-1 $\alpha$ (1:50; Novus Biologicals), rabbit anti-GFAP (1:200; Abcam), rabbit anti-NeuN (1:50; CST, USA), mouse anti-CC1 (1:100; Millipore, USA), rabbit anti-Olig2 (1:1,000; Novus Biologicals), and mouse anti-CS-56 (1:100; Sigma, USA). The following day, the sections were washed and incubated for $1 \mathrm{~h}$ at room temperature with secondary antibodies against the appropriate species: donkey anti-mouse IgG Cy2 (1:500; Jackson ImmunoResearch, USA), goat anti-rabbit IgG Cy3 (1:500; Jackson ImmunoResearch, USA), and goat anti-mouse IgM 488 (1:500; Abcam, UK). Fluorescence images were collected using a confocal laser microscope system (TCS-SP8, Leica). ImageJ (US National Institutes of Health) was used for quantitative analysis of fluorescent signals.

\section{Electron microscopy}

At P42, rats were deeply anaesthetized and perfused briefly with saline followed by glutaraldehyde (BP0130, Bios Biology, China). Whole brains were embedded in fixative 
(G1102, Servicebio, China) at $4{ }^{\circ} \mathrm{C}$ for $4 \mathrm{~h}$, and the right corpus callosum was dissected carefully and fixed in fresh fixative overnight at $4{ }^{\circ} \mathrm{C}$. Ultrathin sections were doublestained with uranyl acetate and lead citrate for electron microscopy imaging. For analysis of the myelination of the corpus callosum, the outer axon parameter was divided by the parameter of the associated myelin sheath to calculate the myelin g-ratios in cross-sections of the corpus callosum. High ratios indicate hypomyelination. Measurements were made on electron micrographs using ImageJ and the g-ratio plug-in (http://www.gratio.efil.de/) from 4-6 rats per group (at least 50 myelinated axons per animal).

\section{Behavioural testing}

At P35, before any of the behavioural tests, all rats were familiarized with the testing environment and investigator for 1 week to eliminate the effect of nonspecific irritation. Morris water maze test includes navigation trials and space probe trials to examine learning and memory function. A navigation trial was performed from $\mathrm{P} 43$ to $\mathrm{P} 46$ in a specific pool with the water depth remaining $1 \mathrm{~cm}$ above the platform. The swimming distance and latency to board the platform from the 4 quadrants of the pool were assessed, and the mean distance and latency of each rat per day were recorded. A space probe trial was performed at $\mathrm{P} 48$, and each rat was placed in the water in the farthest quadrant from the platform after removing the platform. The swimming path of each rat for $30 \mathrm{~s}$ after entering the water was analysed to assess the percentage of time in the target quadrant and the number of times the animal crossed the old platform.

\section{Statistical analysis}

Raw data from ImageJ analyses and behavioural tests were imported into SPSS Statistics 20.0 (IBM, USA) for statistical analyses using Student's $t$-test or ANOVA. The results are expressed as the mean \pm SEM. Graphs were produced in Prism 8. A value of $\mathrm{P}<0.05$ was considered statistically significant.

\section{Results}

\section{Brain tissue injury and abnormal myelination in the rat model of P3 HI injury}

Initially, histopathological analyses of haematoxylin and eosin (H-E)-stained brain sections were performed at $14 \mathrm{~d}$ post-HI (P17) (Figure 1A). We found a disorganized structure and decreased cell density in the corpus callosum area of the HI-exposed ipsilateral side. To determine whether the observed structural change was caused by abnormal myelination rather than neuronal degeneration, we performed MBP (mature oligodendrocyte marker) and NFH (mature neuron marker) double staining on brain sections $14 \mathrm{~d}$ post-HI. As expected, we found that the signal intensities of MBP but not NFH in the ipsilateral corpus callosum decreased (Figure 1B), and the ratio of MBP/NFH significantly declined (Figure $1 C$ ) in the $\mathrm{HI}$ injury group, indicating hypomyelination $(0.929 \pm 0.086$ vs. $0.502 \pm 0.068$, $\mathrm{P}=0.039$ ).

\section{Temporal pattern of HIF-1 $\alpha$ expression after HI injury}

To explore the temporal expression of HIF- $1 \alpha$ in the ipsilateral corpus callosum after HI injury, we performed western blotting at $12 \mathrm{~h}, 24 \mathrm{~h}, 36 \mathrm{~h}, 72 \mathrm{~h}, 7 \mathrm{~d}$, and $14 \mathrm{~d}$ post$\mathrm{HI}$ injury. We found that the expression of HIF- $1 \alpha$ increased from $12 \mathrm{~h}$ (by 1.49 times) to $24 \mathrm{~h}$ (by 2.11 times) postinjury, decreased quickly at $36 \mathrm{~h}$ post-HI, was upregulated again from $72 \mathrm{~h}$ to $7 \mathrm{~d}$ (by 2.14 times), and declined to the control level at $14 \mathrm{~d}$ post-injury $(\mathrm{P}=0.027$ at $12 \mathrm{~h}, 0.009$ at $24 \mathrm{~h}$, and $<0.001$ at $7 \mathrm{~d}$ ) (Figure $2 A$ ). Immunofluorescence was performed to further investigate the temporal pattern of HIF- $1 \alpha$ expression. We found that HIF- $1 \alpha$ could barely be detected in the control group (Figure $2 B$ ). In accordance with Figure $2 A$, we found that the signal intensities of HIF- $1 \alpha$ in the HI-exposed ipsilateral corpus callosum at $24 \mathrm{~h}$ and $7 \mathrm{~d}$ post-HI injury were significantly elevated $(\mathrm{P}=0.024$ at $24 \mathrm{~h}$, and 0.024 at $7 \mathrm{~d}$ ) (Figure $2 C, 2 D)$. Double staining was performed to evaluate the sources of HIF- $1 \alpha$. We found that HIF- $1 \alpha$ colocalized with NeuN (+) neurons instead of Olig2 (+) oligodendrocytes or GFAP (+) astrocytes at $24 \mathrm{~h}$ post-HI injury (Figure $2 E$ ), while HIF$1 \alpha$ expression appeared in astrocytes at $72 \mathrm{~h}$ post-HI injury and mainly colocalized with astrocytes at $7 \mathrm{~d}$ post-HI injury (Figure 2F,2G). These results suggested that the effect of the HIF-1 $\alpha$ increase at different time points after $\mathrm{HI}$ injury in the ipsilateral corpus callosum should be different.

\section{Effect of delayed HIF-1 $\alpha$ increase on myelination and gliosis}

To investigate the effect of delayed increase in HIF- $1 \alpha$ 
A
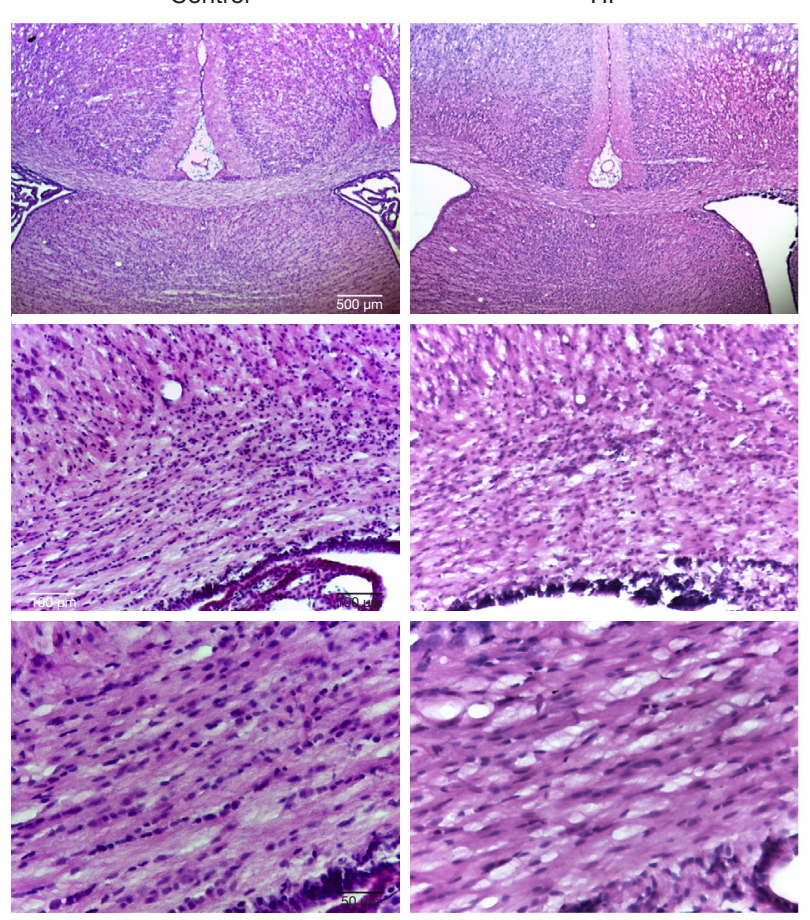

B
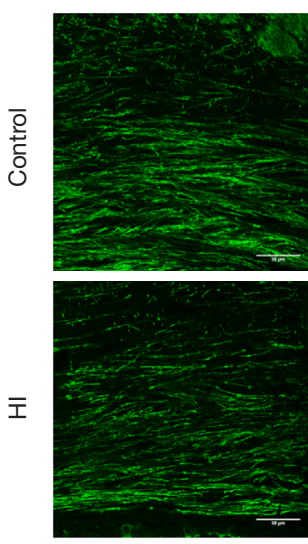

C

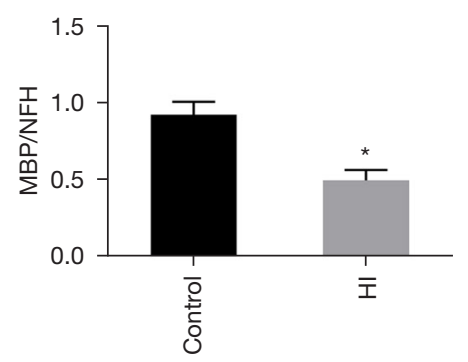

MBP/NFH
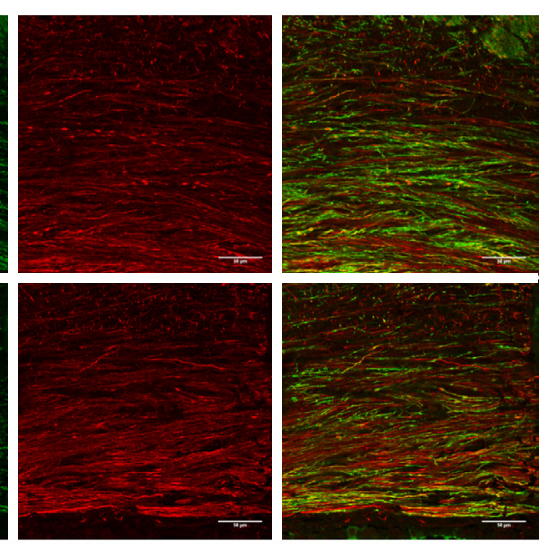

Figure 1 Brain tissue injury and abnormal myelination in the P3 HI injury rat model. (A) Representative pictures of H-E staining in the ipsilateral corpus callosum at $14 \mathrm{~d}$ post injury (P17). Scale bar: $500 \mu \mathrm{m}$ (top), $100 \mu \mathrm{m}$ (middle), $50 \mu \mathrm{m}$ (bottom). (B) Representative pictures of immunofluorescence stained with MBP (green) and NFH (red) in the ipsilateral corpus callosum at $14 \mathrm{~d}$ post injury (P17). Scale bar: $50 \mu \mathrm{m}$. (C) The ratio of the MBP and NFH signal intensities. Values were shown as mean \pm SEM; $n=3-4$ /group; * $\mathrm{P}<0.05$ by $t$-test.

after HI injury, we inhibited HIF-1 $\alpha$ using 2-ME at $72 \mathrm{~h}$ post-injury (Figure $3 A$ ) and found the expression of HIF$1 \alpha$ at 7 days post injury decreased significantly (Figure $3 B$ ). Myelination, oligodendrocyte maturation, and gliosis were assessed at 14 days post-HI (P17) by western blots and immunofluorescence (Figure $3 A$ ). We found the expression of $\mathrm{MBP}$ increased dramatically in the late HIF- $1 \alpha$ inhibition group $(\mathrm{H}+\mathrm{M} 3)$ at $14 \mathrm{~d}$ post-HI injury compared to the blank HI group $(\mathrm{H}+\mathrm{B} 3)(0.941 \pm 0.067 v s$. $0.632 \pm 0.083, \mathrm{P}=0.033$ ) (Figure $3 C$ ). The increase in MBP expression in the late inhibition group was also proven by immunofluorescence $(0.899 \pm 0.052$ in $\mathrm{H}+\mathrm{M} 3$ vs. $0.509 \pm 0.068$ in $\mathrm{H}+\mathrm{B} 3, \mathrm{P}=0.021)$, indicating the improvement of myelination (Figure 3D). In addition, we found that the percentage of CC1 (+) Olig2 (+) mature oligodendrocytes in the Olig2 (+) oligodendrocytes in the late HIF-1 $\alpha$ inhibition group $(87.50 \%)$ was significantly higher than that in the HI group $(70.40 \%)(\mathrm{P}<0.001)$, indicating that late HIF-1 $\alpha$ inhibition could improve oligodendrocyte maturation
(Figure 3E). Since late-onset HIF-1 $\alpha$ expression mainly appeared in astrocytes, we hypothesized that it should be involved in reactive gliosis after immature white matter injury. Double staining with CS-56 (anti-chondroitin sulfate antibody) and GFAP (Figure $4 A$ ) revealed more astrocyte activation $(1,403 \pm 129$ vs. $805 \pm 69, \mathrm{P}=0.019$, Figure $4 B)$ and gliosis $(36,969 \pm 2,632$ vs. $21,180 \pm 601, \mathrm{P}=0.004$, Figure $4 C)$ in the HI-exposed ipsilateral corpus callosum compared to those in the control group. However, the expression of GFAP (791 \pm 121 vs. $1,403 \pm 129, \mathrm{P}=0.017$, Figure $4 B)$ and CSPGs $(20,909 \pm 1,782$ vs. $36,969 \pm 2,632, \mathrm{P}=0.006$, Figure $4 C$ ) decreased dramatically in the late inhibition group in contrast to the blank HI group, suggesting a late HIF- $1 \alpha$ increase in astrocytes involved in astroglial activation and reactive gliosis after $\mathrm{HI}$ white matter injury. Combined with our previous studies (15), these results suggested that the improvement in myelination and oligodendrocyte maturation after late inhibition of HIF-1 $\alpha$ could be partly related to reduced reactive gliosis. 
A

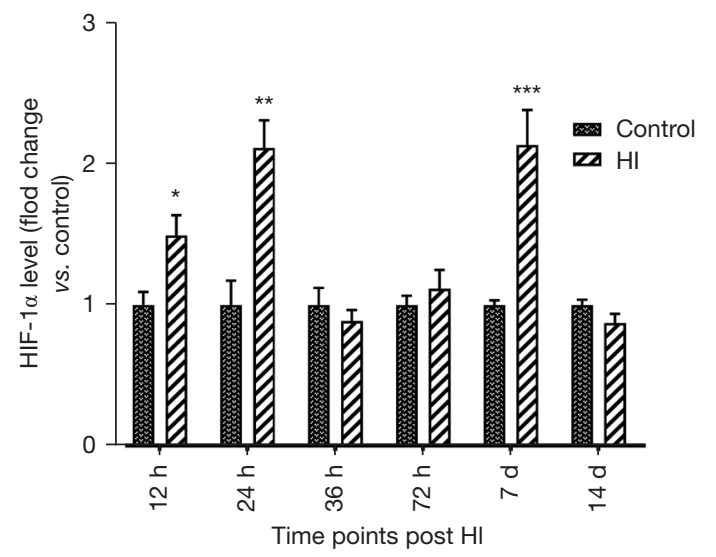

E
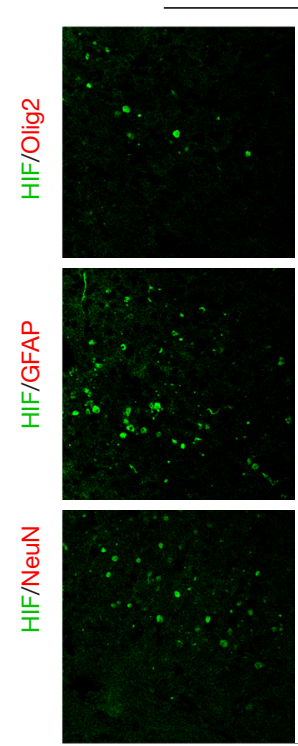

$24 \mathrm{~h}$ post $\mathrm{HI}$
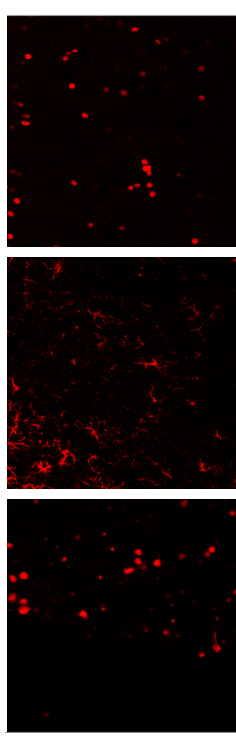

B

$24 \mathrm{~h}$
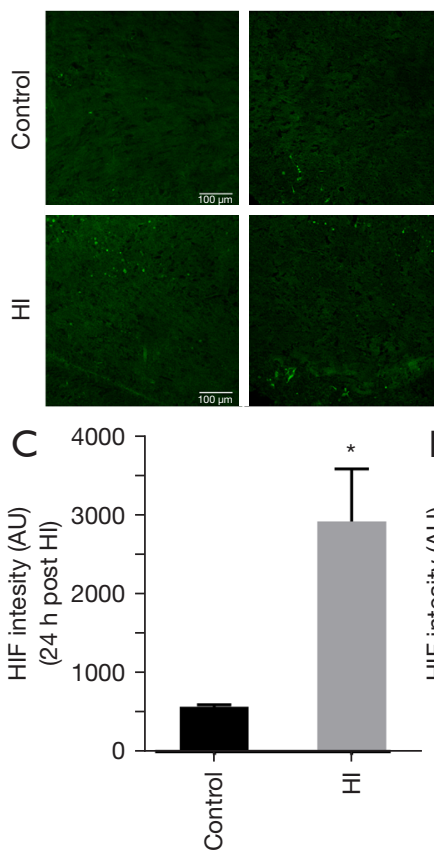

$7 \mathrm{~d}$
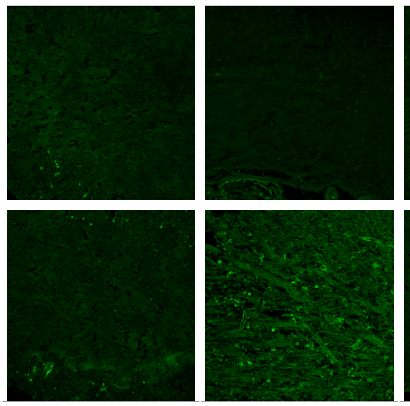

D 15000

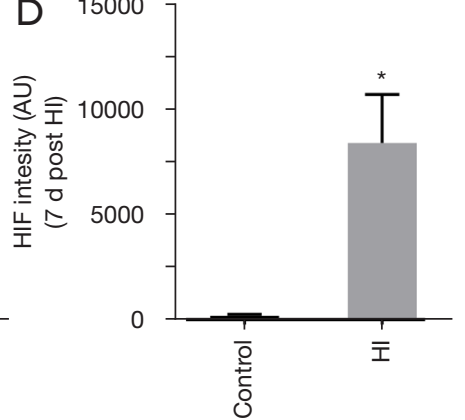

$\mathrm{F}$

$72 \mathrm{~h}$ post $\mathrm{HI}$
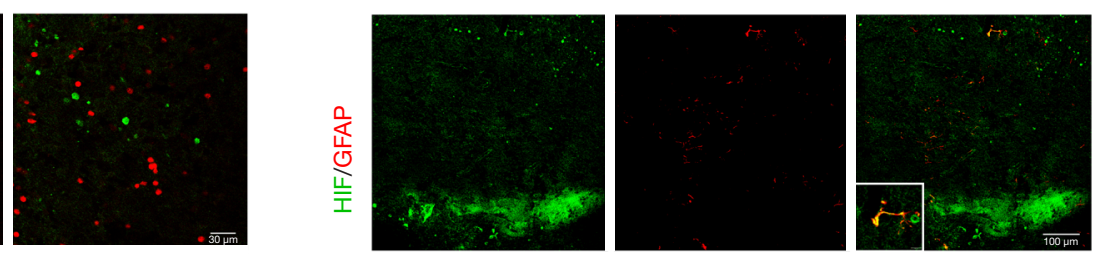

G

$7 \mathrm{~d}$ post $\mathrm{HI}$
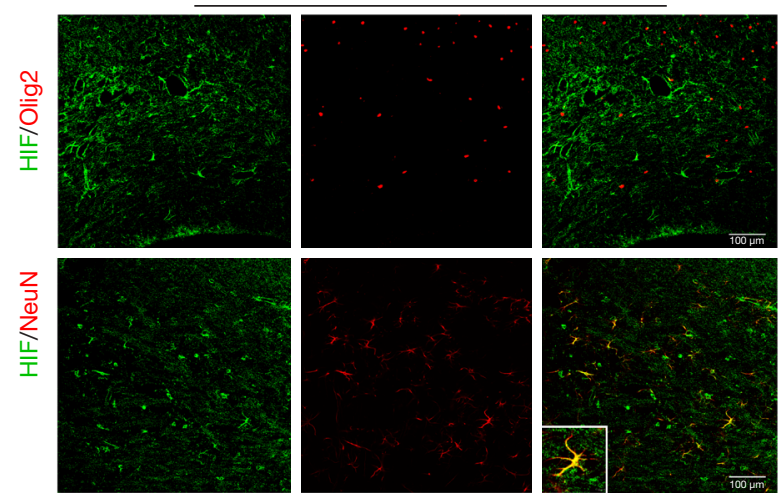

Figure 2 Temporal pattern of HIF-1 $\alpha$ expression in the ipsilateral corpus callosum after HI injury. (A) Western blot quantification of HIF-1 $\alpha$ protein in the ipsilateral corpus callosum at different time points post $\mathrm{HI}$ injury. $\mathrm{n}=6-8$ /group. (B) Representative pictures of immunofluorescence of HIF- $1 \alpha$ in the ipsilateral corpus callosum at 24 h, 72 h, 7 d, and 14 d post HI injury. (C,D) Quantification of HIF-1 $\alpha$ intensities at $24 \mathrm{~h}$ and $7 \mathrm{~d}$ post $\mathrm{HI}$ injury. (E-G) Representative pictures of immunofluorescence stained with anti-HIF-1 $\alpha$ (green) and antiOlig2 (red), anti-GFAP (red) or anti-NeuN (red) antibodies in the ipsilateral corpus callosum at $24 \mathrm{~h}, 72 \mathrm{~h}$, and $7 \mathrm{~d}$ post $\mathrm{HI}$ injury. Scale bars in (B): $100 \mu \mathrm{m}$; in (E): main images, $30 \mu \mathrm{m}$, insets $5 \mu \mathrm{m}$; in (F) and (G): main images, $100 \mu \mathrm{m}$, insets, $10 \mu \mathrm{m}$; $\mathrm{n}=3-4 /$ group. Values were shown as mean $\pm \mathrm{SEM} ;{ }^{*}, \mathrm{P}<0.05 ;{ }^{* *}, \mathrm{P}<0.01$; ${ }^{* *}, \mathrm{P}<0.001$ by $t$-test. 

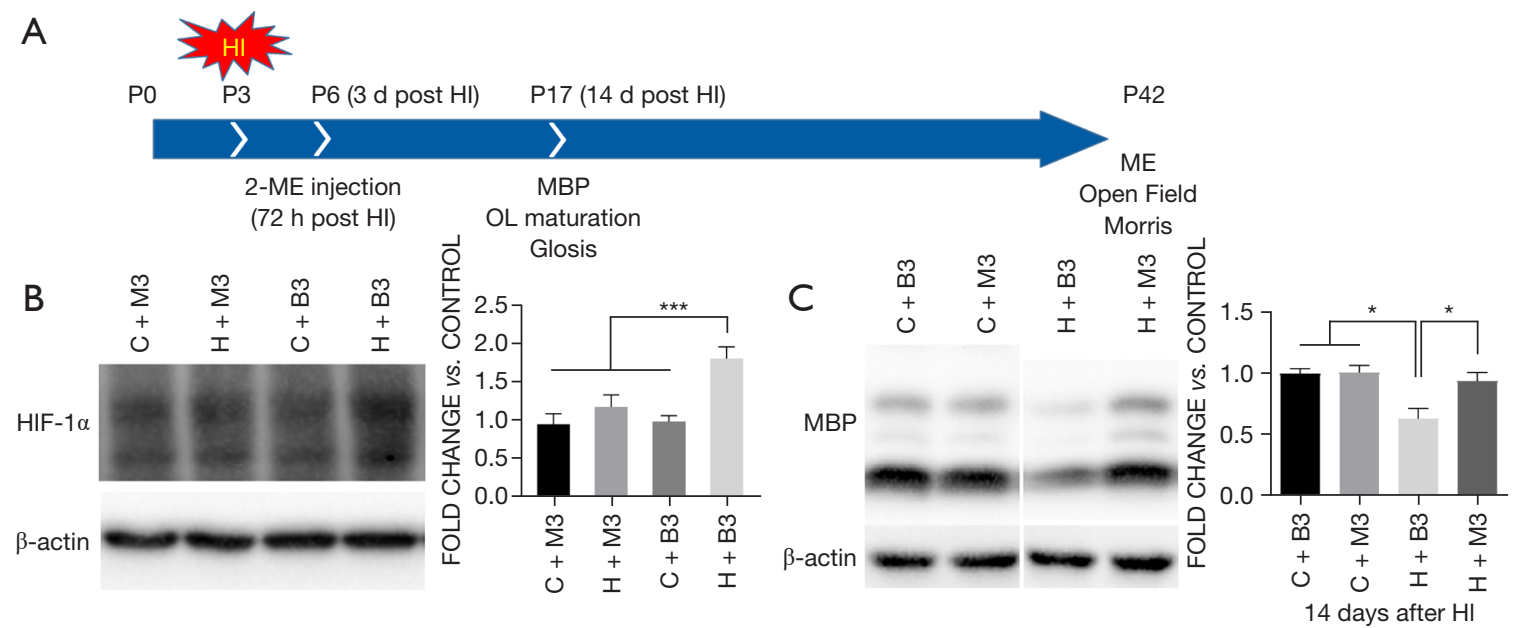

D
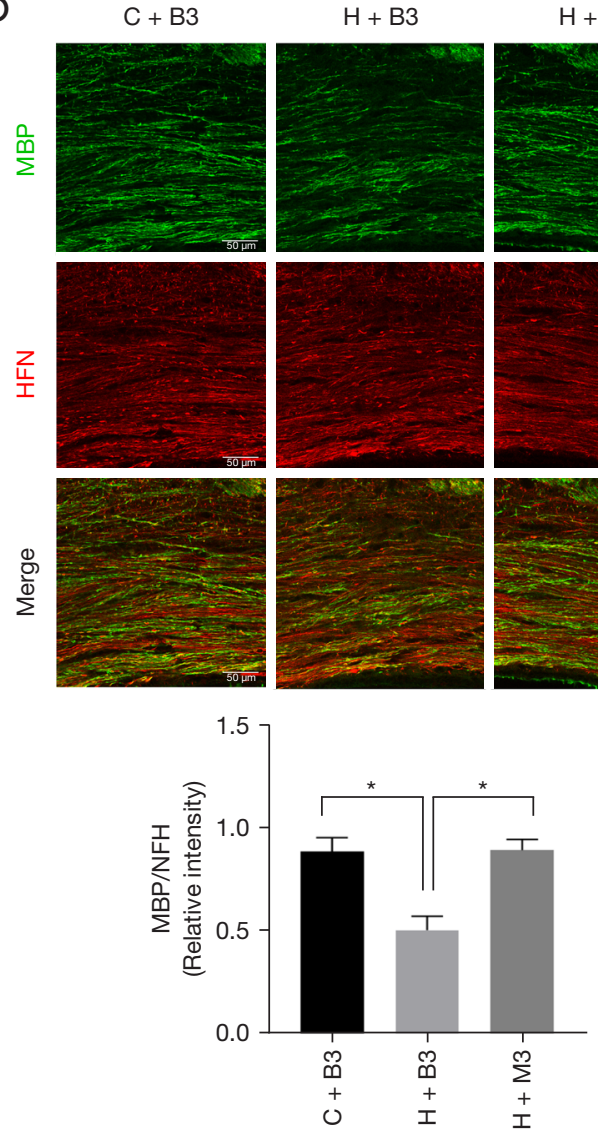

$\mathrm{H}+\mathrm{M} 3$
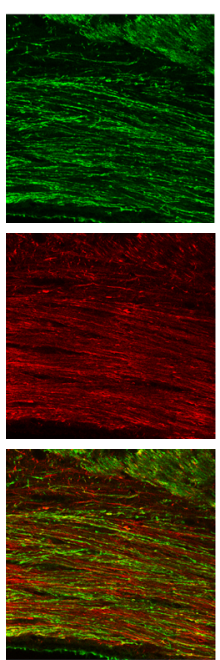

E

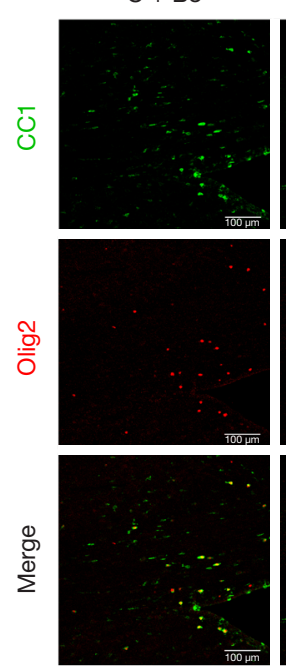

$\mathrm{H}+\mathrm{B} 3$
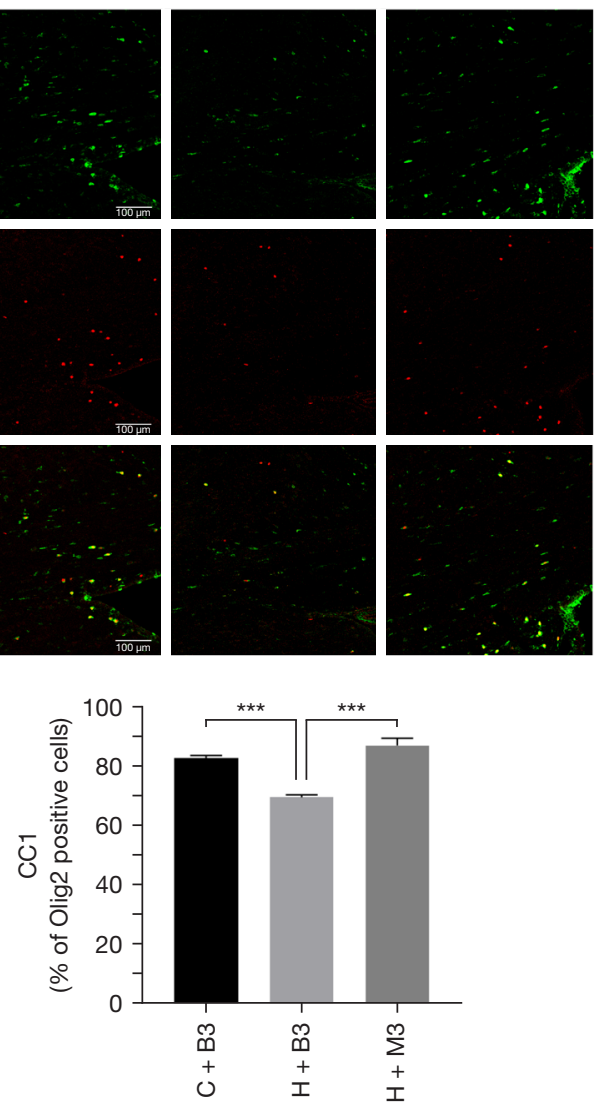

Figure 3 Effect of late-onset HIF-1 $\alpha$ increase on myelination. (A) Timeline of the experimental paradigm of HI injury, intervention, and detection. (B) Representative western blot image and quantification of HIF-1 $\alpha$ expression at 7 days post injury after 2-ME injection. (C) Western blot images and quantification of MBP on $14 \mathrm{~d}$ post injury after late HIF-1 $\alpha$ inhibition. n=8-10/group. (D) Representative pictures of immunofluorescence stained with MBP (green) and NFH (red) in the ipsilateral corpus callosum and the ratio of MBP and NFH signal intensities at $14 \mathrm{~d}$ post injury after late HIF-1 $\alpha$ inhibition. (E) Representative pictures of immunofluorescence stained with CC1 (mature oligodendrocyte marker) (green) and Olig2 (oligodendrocyte marker) (red) in the ipsilateral corpus callosum and the percentage of CC1 (+) Olig2 (+) cells of Olig2 (+) cells at $14 \mathrm{~d}$ post injury after late HIF-1 $\alpha$ inhibition. Scale bar: $100 \mu \mathrm{m} ; \mathrm{n}=3-4 /$ group. Values were shown as mean \pm SEM; *, $\mathrm{P}<0.05$; *** $\mathrm{P}<0.001$ by ANOVA followed by Tukey's post hoc test. $\mathrm{C}+\mathrm{B} 3$ : control with blank injection group, $\mathrm{C}+\mathrm{M} 3$ : control with 2-ME injection group, $\mathrm{H}+\mathrm{B} 3$ : $\mathrm{HI}$ injury with blank injection group, $\mathrm{H}+\mathrm{M} 3$ : $\mathrm{HI}$ injury with 2-ME injection group. 


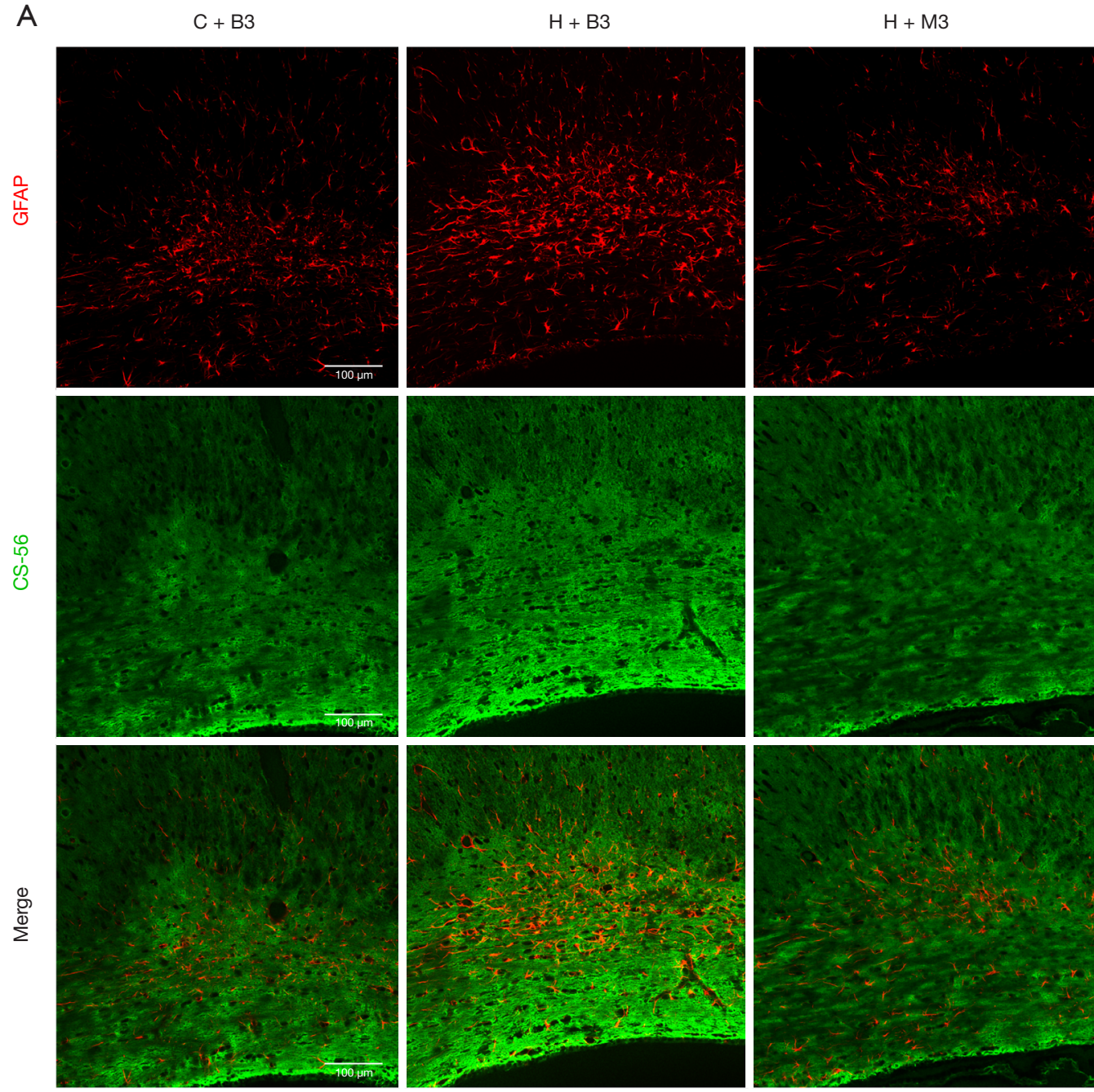

B

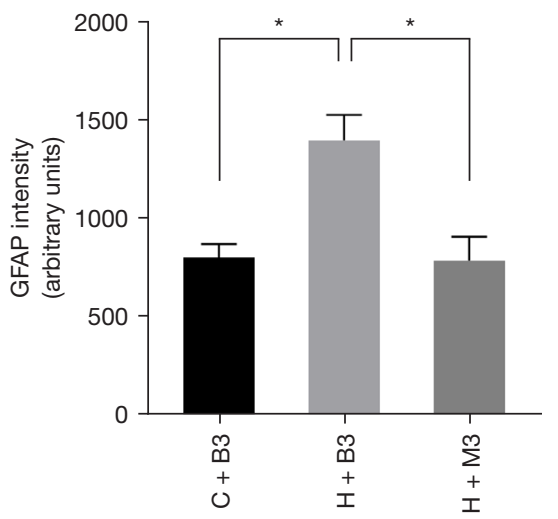

C

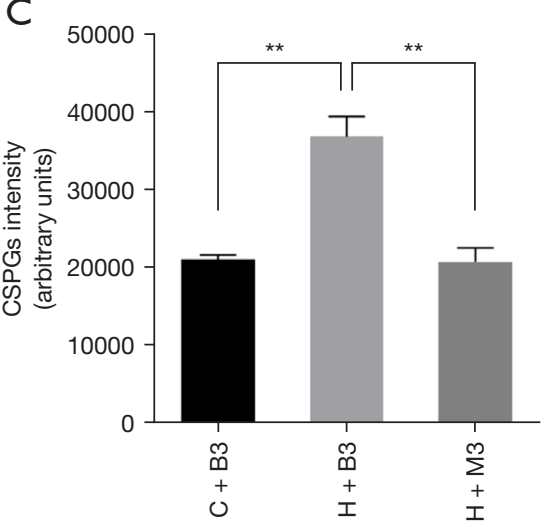

Figure 4 Effect of late-onset HIF-1 $\alpha$ increase on astrocyte activation and gliosis. (A) Representative picture of immunofluorescence stained with CS-56 (CSPGs marker) (green) and GFAP (red) in the ipsilateral corpus callosum. (B) Quantification of GFAP intensity at $14 \mathrm{~d}$ post injury after late HIF-1 $\alpha$ inhibition. (C) Quantification of CSPGs intensity at $14 \mathrm{~d}$ post injury after late HIF-1 $\alpha$ inhibition. Scale bar: $100 \mu \mathrm{m}$; $\mathrm{n}=3-4$ /group. Values were shown as mean $\pm \mathrm{SEM} ;{ }^{*}, \mathrm{P}<0.05 ;{ }^{* *}, \mathrm{P}<0.01$ by ANOVA followed by Tukey's post hoc test. 


\section{Inbibiting delayed HIF-1 $\alpha$ expression attenuated bypomyelination and ameliorated long-term neurological functions}

Based on the extent of improved oligodendrocyte maturation and reduced gliosis, we hypothesized that late inhibition of HIF-1 $\alpha$ would attenuate abnormal myelination and ameliorate neurological function after $\mathrm{HI}$ injury in the long term. Ultrastructural analysis by electron microscopy of the corpus callosum revealed fewer myelin wraps in the HI group, while myelin appeared more tightly wrapped in the control group and the late inhibition group ( $\mathrm{HI}+\mathrm{M} 3)$ (Figure 5A). Further measurement and analysis of electron micrographs revealed that the myelin sheaths around all sizes of axons, as assessed by the g-ratio and axon parameter, were markedly thicker in the late HIF-1 $\alpha$ inhibition group than in the $\mathrm{HI}$ groups (g-ratio: $0.787 \pm 0.002$ vs. $0.831 \pm 0.002$, $\mathrm{P}<0.001$ ) (Figure $5 B-5 D$ ), indicating improved myelin development.

For analysis of whether the observed myelin structural improvement obtained with late HIF-1 $\alpha$ inhibition translated to ameliorated long-term neurological functions, rats were subjected to Morris water maze tests. Morris water maze test is one of the most widely used tests for the assessment of spatial learning and memory, which are associated with cognitive function. In the navigation trial, rats in each experimental group exhibited learning, as proven by the escape latency and swimming distance decreasing over the training time. The HI group rats displayed long-term learning deficits, as the escape latency and swimming distance were significantly longer than those of the control group, while the late inhibition group had a significantly shorter escape latency $(\mathrm{P}=0.039$ at day $1,0.010$ at day 2, 0.005 at day 3, and 0.046 at day 4) and swimming distance $(\mathrm{P}=0.021$ at day $1,0.008$ at day 2, 0.007 at day 3, 0.039 at day 4) from the first day to the end, than the HI group (Figure 5E, $5 F$ ). After 4 days of training, the rats were assessed in the swimming pool where they were trained for 4 days after withdrawing the underwater platform for memory evaluation. Relative to those in the control group $(42.43 \% \pm 2.56 \%)$, the rats in the $\mathrm{HI}$ group $(28.83 \% \pm 2.29 \%)$ spent less time in the target quadrant $(\mathrm{P}=0.004)$ and had fewer platform crossings $(\mathrm{P}<0.001)$. In contrast, the rats in the late inhibition group $(38.02 \% \pm 3.31 \%)$ displayed much more time in the target quadrant $(\mathrm{P}=0.035)$ and crossings $(2.667 \pm 0.333)$ compared with the HI group $(1.286 \pm 0.421)$ $(\mathrm{P}=0.019)$ (Figure 5G-5H). These results demonstrated that impaired spatial memory and cognitive $\mathrm{HI}$ injury were relieved by late $\mathrm{HIF}-1 \alpha$ inhibition.

\section{Discussion}

We previously demonstrated that CSPGs inhibited both the maturation of oligodendrocytes and the process of myelination after $\mathrm{HI}$ white matter injury. Treatment with cABC could promote myelin formation and cognitive development (15). However, the mechanism of reactive gliosis in white matter injury post-HI remains poorly understood. Here, we tested the expression of HIF-1 $\alpha$ at different time points after HI-evoked white matter injury and found early-onset expression of HIF-1 $\alpha$ in neurons and late-onset expression in astrocytes. In addition, we demonstrated that inhibiting delayed upregulation of HIF- $1 \alpha$ could ameliorate reactive gliosis and improve myelination and cognition after HI-induced white matter injury. These findings help elucidate the role of astrogliosis in HI white matter injury and suggest that inhibiting delayed HIF-1 $\alpha$ elevation may repair this type of lesion.

$\mathrm{HIF}-1 \alpha$ is one of the best-studied transcription factors in various hypoxia- and ischaemia-associated diseases, such as cerebral infarction and myocardial infarction, and controls the expression of a multitude of genes. Due to immature lung and heart development, the brains of preterm infants are more likely to suffer hypoxia and ischaemia, suggesting that HIF-1 $\alpha$ might play an important role in white matter injury in preterm infants. In a 7-day rat model of $\mathrm{HI}$ brain injury, researchers found that the HIF-1 $\alpha$ protein levels in the cortex remained unchanged at $0 \mathrm{~h}$, increased at $24 \mathrm{~h}$, and returned to control levels by $72 \mathrm{~h}$ after HI (22). In addition to hypoxia-dependent HIF-1 $\alpha$ elevation (23), other stress pathways, including the PI3K/Akt pathway (24) and MAPK signalling (25), are involved in HIF-1 $\alpha$ expression. Researchers demonstrated early-onset HIF-1 $\alpha$ elevation in neurons at $24 \mathrm{~h}$ after transient focal cerebral ischaemia in adult mice, while a late-onset increase in astrocytes was initiated at $3 \mathrm{~d}$ and peaked at $7 \mathrm{~d}$ (19). In this research, we found that HIF-1 $\alpha$ expression in the ipsilateral corpus callosum increased from 12 to $24 \mathrm{~h}$ post-HI injury and decreased quickly at $36 \mathrm{~h}$ post-HI, which was consistent with previous studies (22), as an early-onset transient elevation for adaptation to hypoxia. Moreover, there was a significant late-onset elevation of $\mathrm{HIF}-1 \alpha$ at $7 \mathrm{~d}$ post-HI injury. The temporal expression of HIF-1 $\alpha$ suggested that the source of early and late expression of HIF-1 $\alpha$ after HI white matter injury should be different.

In a previous study using a chronic hypoxia neonatal mouse model, HIF-1 $\alpha$ was mainly elevated in oligodendrocytes after 7 days of chronic hypoxia (17). However, double immunofluorescence staining in our 
A Control

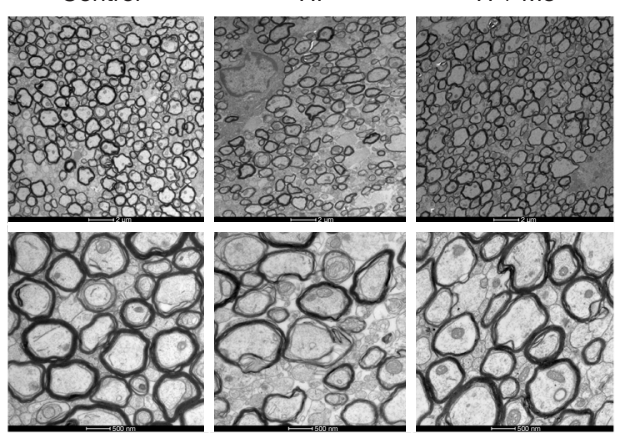

C

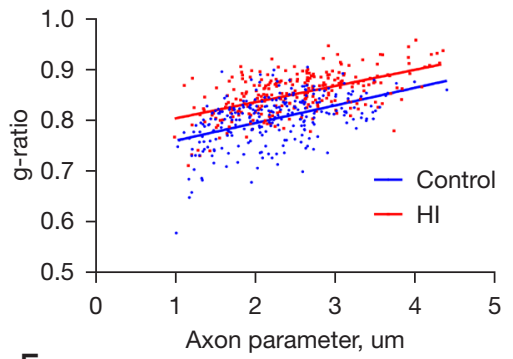

E

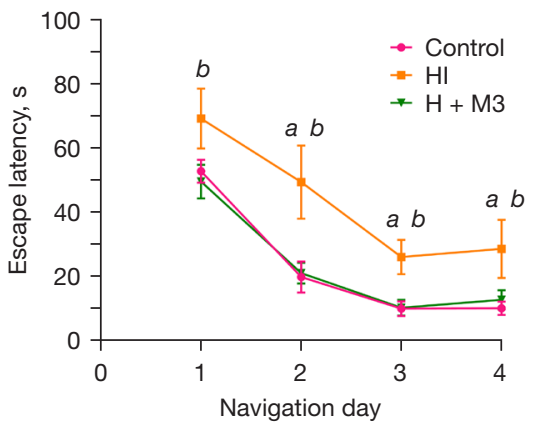

G

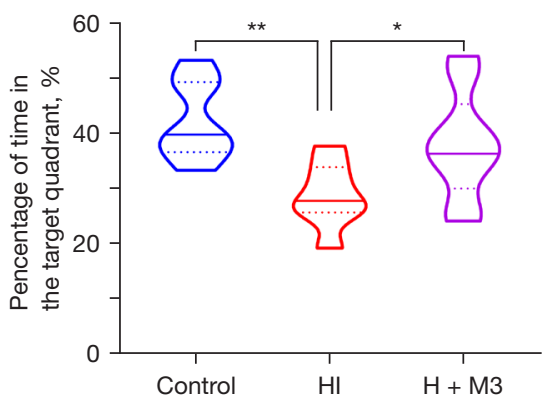

B

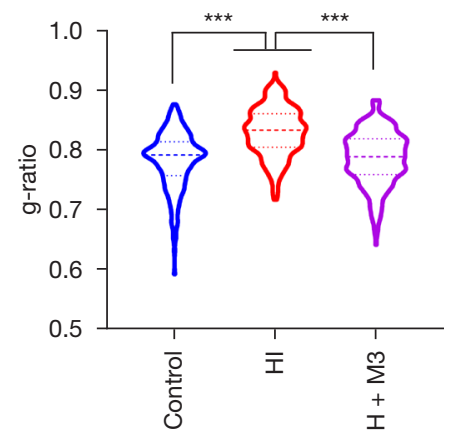

D

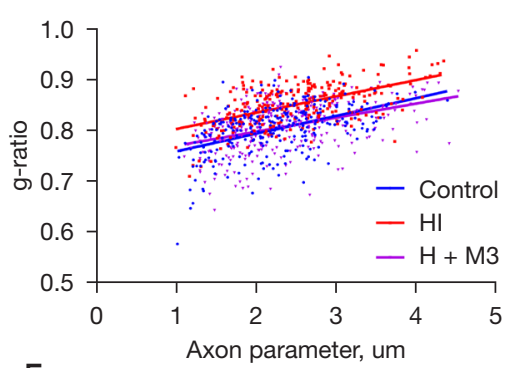

$\mathrm{F}$

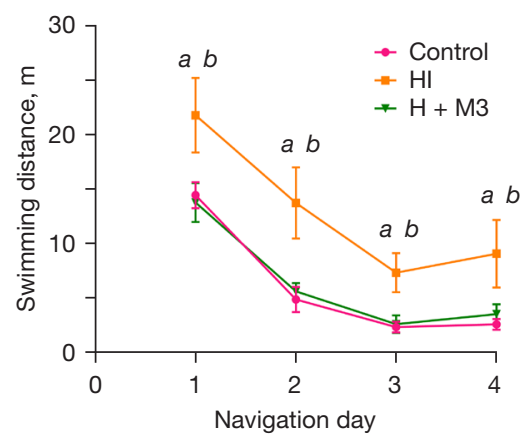

$\mathrm{H}$

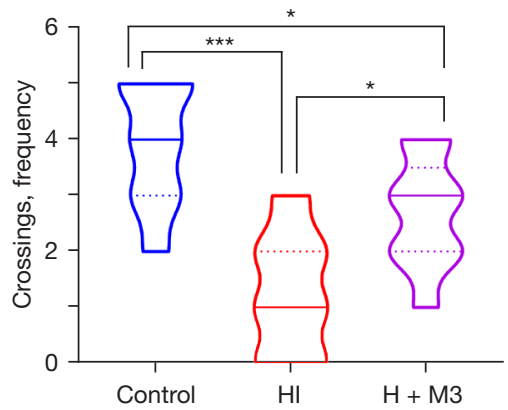

Figure 5 Electron microscopy and behavior function tests. (A) Representative pictures of electron microscopy of corpus callosum. (B) Quantification of G-ratio of myelin. (C and D) Linear regression of G-ratio and axon parameter. (C) The intercepts rather than slopes between control and HI group were significantly different $(\mathrm{P}<0.001)$. (D) The slopes and intercepts between control and late inhibition were not significantly different $(\mathrm{P}>0.05)$, while the intercepts between $\mathrm{HI}$ and late inhibition group were significantly different $(\mathrm{P}<0.001)$. $\mathrm{n}=4-6$ rats/group and at least 50 myelinated axons/rat. Values were shown as mean $\pm \mathrm{SEM}$; ${ }^{* *}, \mathrm{P}<0.001$ by ANOVA followed by Tukey's post hoc test. (E,F) Escape latency and swimming distances of each training day in navigation trail. $a$, significant difference between $\mathrm{HI}$ and control; $b$, significant difference between $\mathrm{HI}$ and late inhibition group. $(\mathrm{G}, \mathrm{H})$ Percentage in the target quadrant and frequency of platform crossings in probe trail. $\mathrm{n}=8$ /group; values were shown as mean $\pm \mathrm{SEM}$;, $\mathrm{P}<0.05 ;{ }^{* *}, \mathrm{P}<0.01$; *** $\mathrm{P}<0.001$ by ANOVA followed by LSD post hoc test. 
study showed that HIF- $1 \alpha$ colocalized with neurons instead of oligodendrocytes or astrocytes at $24 \mathrm{~h}$ post-HI injury, while HIF-1 $\alpha$ mainly colocalized with astrocytes at $7 \mathrm{~d}$ post-HI injury. This difference could be attributed to different species, different sampling time points, and, most importantly, different injury models. The temporal difference in the increase in HIF-1 $\alpha$ between neurons and astrocytes in our study was similar to the results reported by Hirayama et al. (19) using an adult mouse model of cerebral ischaemia, suggesting the different effects of early- and lateonset HIF- $1 \alpha$ elevation in our model.

Many studies have demonstrated the neuroprotection of HIF- $1 \alpha$, including its role in cellular adaptation, recovery and regeneration in response to $\mathrm{HI}$ brain injury (26-28). However, some researchers have found that HIF- $1 \alpha$ is involved in brain oedema, increased blood brain barrier permeability and neuronal apoptosis after injury $(21,29,30)$. The controversial role of HIF- $1 \alpha$ could be due to the different models and the extent of HIF-1 $\alpha$ elevation. In addition to neurons, HIF- $1 \alpha$ also plays important roles in the proliferation, differentiation and activation of oligodendrocytes and astrocytes after brain injury, which is less studied. In a chronic white matter injury model, constitutive HIF- $1 \alpha$ stabilization resulted in OPC maturation arrest and hypomyelination (17). In the present study, inhibiting delayed HIF-1 $\alpha$ elevation significantly improved oligodendrocyte maturation and myelination. Moreover, the rats in the late inhibition group had thicker myelin and improved results in the Morris water maze test, indicating that late HIF-1 $\alpha$ inhibition could attenuate abnormal myelination and ameliorate neurological function after $\mathrm{HI}$ injury in the long term. However, some inconsistencies exist, and late HIF-1 $\alpha$ expression mainly appeared in astrocytes rather than oligodendrocytes (17), suggesting that late HIF-1 $\alpha$ elevation might inhibit OPC maturation and myelination through a paracrine rather than autocrine mode in our model. We found that the $\mathrm{HI}$-exposed rats had worse astrogliosis and more CSPG accumulation, and these responses were less robust with late HIF- $1 \alpha$ inhibition, which was consistent with a previous study using an adult rodent ischaemic model (18). Combined with our previous work, which demonstrated that CSPG accumulation in the white matter regions could result in hypomyelination after HI injury in immature rats (15), the present research indicated that a delayed increase in HIF- $1 \alpha$ in astrocytes could be involved in glial scar formation, leading to arrested oligodendrocyte maturation, impaired myelination and long-term neurological function after experimental white matter injury in immature rats.

However, the mechanism of late-onset HIF-1 $\alpha$ elevation in our model remains unknown. Hypoxia-dependent HIF- $1 \alpha$ elevation occurs in the early period after hypoxia. In addition to hypoxia-dependent mechanisms (23), other stress pathways, including the PI3K/Akt pathway (24) and MAPK signalling (25), are involved in HIF-1 $\alpha$ expression. In a transient focal cerebral ischaemic mouse model, researchers found that a hypoxia-independent but P2X7 receptor-dependent mechanism induced late-onset HIF-1 $\alpha$ expression in astrocytes (19). In future research, we would like to investigate whether the $\mathrm{P} 2 \mathrm{X} 7$ receptor is involved in the late-onset HIF-1 $\alpha$ elevation in astrocytes and could become a therapeutic target in white matter injury.

\section{Conclusions}

In summary, delayed increase in HIF-1 $\alpha$ in astrocytes is involved in glial scar formation, and leads to arrested oligodendrocyte maturation, impaired myelination, and long-term neurological function after experimental white matter injury in immature rats. However, the mechanism of delayed increase in HIF-1 $\alpha$ in astrocytes remains unknown. The next step will be investigate whether the $\mathrm{P} 2 \mathrm{X} 7$ receptor is involved in the late-onset HIF-1 $\alpha$ elevation in astrocytes.

\section{Acknowledgments}

Funding: This work was supported by the National Natural Science Foundation of China (grant number 81671499) and National Key R\&D Program of China (grant number 2017YFA0104200).

\section{Footnote}

Reporting Checklist: The authors have completed the ARRIVE reporting checklist. Available at https:// tp.amegroups.com/article/view/10.21037/tp-21-407/rc

Data Sharing Statement: Available at https://tp.amegroups. com/article/view/10.21037/tp-21-407/dss

Conflicts of Interest: All authors have completed the ICMJE uniform disclosure form (available at https://tp.amegroups. com/article/view/10.21037/tp-21-407/coif). The authors have no conflicts of interest to declare.

Ethical Statement: The authors are accountable for all 
aspects of the work in ensuring that questions related to the accuracy or integrity of any part of the work are appropriately investigated and resolved. Experiments were approved by the Research Ethics Board of the Children's Hospital of Fudan University (No. 2016-06), and rats were treated in accordance with the National Institutes of Health (NIH) Guide for the Care and Use of Laboratory Animals.

Open Access Statement: This is an Open Access article distributed in accordance with the Creative Commons Attribution-NonCommercial-NoDerivs 4.0 International License (CC BY-NC-ND 4.0), which permits the noncommercial replication and distribution of the article with the strict proviso that no changes or edits are made and the original work is properly cited (including links to both the formal publication through the relevant DOI and the license). See: https://creativecommons.org/licenses/by-nc-nd/4.0/.

\section{References}

1. Adams-Chapman I, Heyne RJ, DeMauro SB, et al. Neurodevelopmental Impairment Among Extremely Preterm Infants in the Neonatal Research Network. Pediatrics 2018;141:e20173091.

2. GBD 2017 Causes of Death Collaborators. Global, regional, and national age-sex-specific mortality for 282 causes of death in 195 countries and territories, 1980-2017: a systematic analysis for the Global Burden of Disease Study 2017. Lancet 2018;392:1736-88.

3. Gano D, Andersen SK, Partridge JC, et al. Diminished white matter injury over time in a cohort of premature newborns. J Pediatr 2015;166:39-43.

4. Duerden EG, Halani S, Ng K, et al. White matter injury predicts disrupted functional connectivity and microstructure in very preterm born neonates. Neuroimage Clin 2019;21:101596.

5. Wheelock MD, Austin NC, Bora S, et al. Altered functional network connectivity relates to motor development in children born very preterm. Neuroimage 2018;183:574-83.

6. Wu PM, Shih HI, Yu WH, et al. Corpus callosum and cerebellar vermis size in very preterm infants: Relationship to long-term neurodevelopmental outcome. Pediatr Neonatol 2019;60:178-85.

7. Eikenes L, Løhaugen GC, Brubakk AM, et al. Young adults born preterm with very low birth weight demonstrate widespread white matter alterations on brain DTI. Neuroimage 2011;54:1774-85.
8. Salmaso N, Jablonska B, Scafidi J, et al. Neurobiology of premature brain injury. Nat Neurosci 2014;17:341-6.

9. Buser JR, Maire J, Riddle A, et al. Arrested preoligodendrocyte maturation contributes to myelination failure in premature infants. Ann Neurol 2012;71:93-109.

10. Back SA, Gan X, Li Y, et al. Maturation-dependent vulnerability of oligodendrocytes to oxidative stressinduced death caused by glutathione depletion. J Neurosci 1998;18:6241-53.

11. Jablonska B, Scafidi J, Aguirre A, et al. Oligodendrocyte regeneration after neonatal hypoxia requires FoxO1-mediated p27Kip1 expression. J Neurosci 2012;32:14775-93.

12. Shiow LR, Favrais G, Schirmer L, et al. Reactive astrocyte COX2-PGE2 production inhibits oligodendrocyte maturation in neonatal white matter injury. Glia 2017;65:2024-37.

13. Deng Y, Xie D, Fang M, et al. Astrocyte-derived proinflammatory cytokines induce hypomyelination in the periventricular white matter in the hypoxic neonatal brain. PLoS One 2014;9:e87420.

14. Karus M, Ulc A, Ehrlich M, et al. Regulation of oligodendrocyte precursor maintenance by chondroitin sulphate glycosaminoglycans. Glia 2016;64:270-86.

15. Deng YP, Sun Y, Hu L, et al. Chondroitin sulfate proteoglycans impede myelination by oligodendrocytes after perinatal white matter injury. Exp Neurol 2015;269:213-23.

16. Lee P, Chandel NS, Simon MC. Cellular adaptation to hypoxia through hypoxia inducible factors and beyond. Nat Rev Mol Cell Biol 2020;21:268-83.

17. Yuen TJ, Silbereis JC, Griveau A, et al. Oligodendrocyteencoded HIF function couples postnatal myelination and white matter angiogenesis. Cell 2014;158:383-96.

18. Na JI, Na JY, Choi WY, et al. The HIF-1 inhibitor YC-1 decreases reactive astrocyte formation in a rodent ischemia model. Am J Transl Res 2015;7:751-60.

19. Hirayama Y, Koizumi S. Hypoxia-independent mechanisms of HIF- $1 \alpha$ expression in astrocytes after ischemic preconditioning. Glia 2017;65:523-30.

20. Huang Z, Liu J, Cheung PY, et al. Long-term cognitive impairment and myelination deficiency in a rat model of perinatal hypoxic-ischemic brain injury. Brain Res 2009;1301:100-9.

21. Wu C, Hu Q, Chen J, et al. Inhibiting HIF-1 $\alpha$ by 2ME2 ameliorates early brain injury after experimental subarachnoid hemorrhage in rats. Biochem Biophys Res Commun 2013;437:469-74. 
22. Chu HX, Jones NM. Changes in Hypoxia-Inducible Factor-1 (HIF-1) and Regulatory Prolyl Hydroxylase (PHD) Enzymes Following Hypoxic-Ischemic Injury in the Neonatal Rat. Neurochem Res 2016;41:515-22.

23. Ivan M, Kondo K, Yang H, et al. HIFalpha targeted for VHL-mediated destruction by proline hydroxylation: implications for O2 sensing. Science 2001;292:464-8.

24. Gao N, Shen L, Zhang Z, et al. Arsenite induces HIF1alpha and VEGF through PI3K, Akt and reactive oxygen species in DU145 human prostate carcinoma cells. Mol Cell Biochem 2004;255:33-45.

25. Sang N, Stiehl DP, Bohensky J, et al. MAPK signaling upregulates the activity of hypoxia-inducible factors by its effects on p300. J Biol Chem 2003;278:14013-9.

26. Sheldon RA, Osredkar D, Lee CL, et al. HIF-1 alpha-

Cite this article as: Wang $\mathrm{MJ}$, Li $\mathrm{ZH}$, Gao RW, Chen QF, Lin J, Xiao ML, Zhang K, Chen C. Effects of delayed HIF-1 $\alpha$ expression in astrocytes on myelination following hypoxiaischaemia white matter injury in immature rats. Transl Pediatr 2022;11(1):20-32. doi: 10.21037/tp-21-407 deficient mice have increased brain injury after neonatal hypoxia-ischemia. Dev Neurosci 2009;31:452-8.

27. Cunningham LA, Candelario K, Li L. Roles for HIF-1 $\alpha$ in neural stem cell function and the regenerative response to stroke. Behav Brain Res 2012;227:410-7.

28. Khan M, Dhammu TS, Baarine M, et al. GSNO promotes functional recovery in experimental TBI by stabilizing HIF-1 $\alpha$. Behav Brain Res 2018;340:63-70.

29. Chen W, Jadhav V, Tang J, et al. HIF-1alpha inhibition ameliorates neonatal brain injury in a rat pup hypoxicischemic model. Neurobiol Dis 2008;31:433-41.

30. Li A, Sun X, Ni Y, et al. HIF-1 $\alpha$ involves in neuronal apoptosis after traumatic brain injury in adult rats. $\mathrm{J}$ Mol Neurosci 2013;51:1052-62. 